Article

\title{
Association of Family Type with Happiness Attributes Among Older Adults: 2017 Korean Community Health Survey Analysis
}

\author{
Eun Jeong Hwang ${ }^{1}$ and In Ok Sim ${ }^{2}$ \\ 1 Sehan University; 1113 Samho-eup, Yeongam-gun, Jeollanam-do, 58447, Republic of Korea; \\ ejhwang@sehan.ac.kr \\ 2 Red Cross College of Nursing, Chung-Ang University, 84 Heukseok-ro, Dongjak-Gu, Seoul 156-756, \\ Republic of Korea; hiraly@cau.ac.kr \\ * Correspondence: hiraly@cau.ac.kr; Tel.: +82-10-3372-5920, Fax: +82-2-824-7961,
}

\begin{abstract}
This study used secondary data from the 2017 Korean Community Health Survey to compare the associations of socio-physical environment, social support networks, and social activities with happiness among older adults with three different family types. As per the results, there was a significant difference in happiness index between the three groups $(\mathrm{F}=86.688, \mathrm{p}<.001)$. Older adults living alone (odds ratio $(\mathrm{OR})=0.75,95 \%$ confidence interval $(\mathrm{CI})=0.57-0.99)$ and those living with family $(\mathrm{OR}=0.80,95 \% \mathrm{CI}=0.65-0.99)$ showed greater happiness as the frequency of contact with family increased. Older adults living with only their spouse showed an increase in happiness when their contact with friends was higher $(\mathrm{OR}=0.69,95 \% \mathrm{CI}=0.56-0.84)$. It was confirmed that there were differences in factors influencing happiness according to the family type of the older adults in this study. Therefore, the happiness of older adults needs to be adequately ensured, while considering their various circumstances, including family type.
\end{abstract}

Keywords: older adult; happiness; family type; socio-physical environment; social activities

\section{Introduction}

In South Korea, the proportion of people aged 65 years and older was $7.03 \%$ of the total population, and Korea became an aging society in 2000 [1]. Subsequently, in 2018, South Korea went on to become an aged society, with the proportion of people aged 65 years and older standing at $14.76 \%$ of the total population. With this rapid aging rate, older adults' family types have changed in various ways. In particular, the proportion of older adults living alone has increased, from $16 \%$ in 2000 to $19.1 \%$ in 2017 . The number of older adults living alone is expected to more than double by 2035 [2].

Families should be among the major sources of support for older adults. The happiness of older adults is likely to be realized when they receive physical care, emotional support, and financial assistance from their families, and are connected with community networks [3]. Older adults are vulnerable to loneliness, which has a significant correlation with poor income, low educational level, living alone, low quality of social relationships, poor self-reported health, and poor functional status [4]. Older adults living alone are likely to have significantly lower psychological well-being, selfesteem, and life satisfaction than older adults living with others [5]. Many older adults living alone are likely to be represented as unhealthy, lacking in vitality or a feeling that living is worthwhile, and lacking pride as members of society [6]. Among older adults, feelings of happiness differ according to their family types. 
Happiness is a universal value, and the Korean Constitution guarantees the right to pursue it [7]. In the past, growth-oriented societies considered income and happiness proportional to each other and used gross domestic product as an alternative measure of happiness. However, economic growth has little effect on happiness, as shown by the Easterlin Paradox [8]. Therefore, globally, people have become more interested in happiness as an outcome in itself. The Organization for Economic Cooperation and Development [9] suggests a "Better Life Index" system. In particular, people should pay attention to the happiness of older adults because as the proportion of older adults in the total population increases, the influence of their happiness on the overall happiness of society will also increase. It would be difficult to realize national happiness if older adults are not happy.

Happiness comprises many similar concepts, which include well-being, quality of life, subjective well-being, life satisfaction, and so on [10]. Studies have suggested various influencing factors regarding the happiness of older adults. In the Madrid International Plan of Action on Ageing, the United Nations [11] recommended three directions for contributing to society through vibrant aging: focusing on older persons and development, advancing health and well-being into old age, and ensuring enabling and supportive environments. The World Health Organization (WHO) [12] presented a global age-friendly city guide for happy and active aging, which includes living environment, transportation, social participation, community support, and health services. The Korea Institute for Health and Social Affairs (KIHSA) [3] presented various factors influencing quality of life in older adults, such as subjective satisfaction, relationships, community, natural environment, health, housing, and safety. Happiness is a subjective feeling that is achievable when various criteria are satisfied. Therefore, measuring happiness requires a comprehensive approach including community environment, housing and safety, and social relationships.

In particular, considering differences in the degree of support that older adults receive from their families, strategies should be devised to enable a healthy and happy life using various social resources. Identifying the factors influencing older adults' happiness according to their family type and finding ways to improve their happiness is, thus, important. Specifically, the WHO [12] has covered major factors influencing happiness identified in previous studies. Green and Kreuter's [13] PRECEDE-PROCEED model provides an excellent explanation of the relationships between behavior and lifestyle factors, environmental factors, health, and quality of life. It is used as a comprehensive conceptual framework to change health levels and quality of life by improving health behaviors and environment for various groups in the community [14]. This model explains that not only an individual's behavior and attitude but also the community environment affects health and quality of life [15]. It emphasizes that behavioral, social, and ecological factors have a profound impact on health and quality of life.

In the present study, the factors associated with older adults' happiness were derived from the PRECEDE-PROCEED model and a literature review. The Korean government has been working to promote the health and quality of life of older adults for decades. It is important to verify the results of these government efforts and seek ways to improve them by family type.

\subsection{Purpose}

This study compared the associations of present health status, depressive symptoms, social and physical environment, social support networks, and social participation with happiness among older adults living alone, with only their spouse, or with their families. Ultimately, this study provides useful information for the development of happiness programs that consider older adults according to their family types.

\section{Materials and Methods}

\subsection{Design}

The study employed a non-experimental, correlational research design. 


\subsection{Data Collection and Procedures}

The secondary data used in this study were extracted from the 2017 Community Health Survey conducted by the Korea Centers for Disease Control and Prevention (KCDC). These data are available free of charge for research purposes. These annual surveys, conducted nationwide, utilize the public health center network. The sample selection for the 2017 survey was set up as a target population for adults aged 19 and over. Finally, all the sample households were included in the survey. In the data collection process, trained researchers visited the selected households and explained the purpose and confidentiality of the survey to the respondents, and administered an electronic questionnaire through a one-to-one interview. The survey period was from August 16 to October 31, 2017. For the present study, 14,687 subjects aged 65 years or older were selected from the 2017 Community Health Survey data and subsequently divided into three groups: older adults living alone, older adults living with their spouse, and older adults living with their families. The classification criteria were as follows: one-person households were classified as older adults living alone, two-person households consisting of couples were classified as older adults living with a spouse, and if the number of household members was two or more but they were not a couple, they were classified as older adults living with their families.

\subsection{Dependent Variable}

The happiness index of older adults consisted of one item, which was measured on a 10-point Likert scale, with higher scores indicating greater happiness. For the purpose of binary logistic regression analysis, on the basis of the happiness index results, older adults were divided into two categories: unhappy (score 1-5) and happy (score 6-10).

\subsection{Independent Variables}

The independent variables were five factors derived from the WHO [12] active aging guide: depressive symptoms, present health status, satisfaction with socio-physical environment, social support networks, and participation in social activities.

\subsubsection{Depressive symptoms}

These were measured with nine items: "no interest in or fun at work," "sinking feeling, depression, and hopelessness," "difficulty getting to sleep or sleeping too much," "feeling tired," "lack of appetite or overeating," "considering oneself worthless and a harbinger of misery," "difficulty concentrating on newspapers or television," "nervousness, anxiety, or too much wandering," and "believing that death is preferable to living or thoughts about hurting oneself." Each item was measured on a four-point Likert scale $(1=$ never, $2=$ felt for several days, $3=$ felt for over a week, 4 = felt almost every day), with higher scores indicating more depression. Cronbach's $\alpha$ of the depression instrument was 0.83 .

\subsubsection{Present health status}

This was measured with five items: athletic ability, self-management, daily activities, pain and discomfort, and anxiety. Each item was measured on a three-point scale $(1=$ bad, $2=$ somewhat bad, $3=$ good), with higher scores indicating better levels of health. Cronbach's $\alpha$ of the health status instrument was 0.83 .

\subsubsection{Satisfaction with socio-physical environment}

This was measured with seven items: "trust in neighbors," "help from neighbors," "neighborhood safety level (disasters, traffic accidents, work accidents, crime)," "natural environment (air and water quality)," "life environment (electricity, water, sewage, garbage collection, athletic facilities)," "condition of public transportation (buses, taxis, trains, subway)," and "condition of medical facilities (hospitals, community health centers, oriental hospitals, 
pharmacies)." Each item was measured on a dichotomous scale $(1=$ dissatisfied, $2=$ satisfied $)$. The Kuder-Richardson Formula 20 value of the socio-physical environment instrument was 0.60.

\subsubsection{Social support networks}

These were assessed with three items: "contact with family (or relatives)," "contact with neighbors," and "contact with friends." Each item was measured on a six-point Likert scale $(1=$ less than once per month, $2=$ once a month, $3=$ two or three times per month, $4=$ once a week, $5=$ two or three times a week, 6 = four or more times a week).

\subsubsection{Participation in social activities}

This was assessed with four items: "religious activities," "belonging to communities," "leisure activities," and "charity activities." Each item was measured on a dichotomous scale $(1=$ nonparticipation, 2 = participation).

\subsection{Ethical Considerations}

The KCDC provides the data used in this study free of charge for research purposes subsequent to certain official procedures and approval. The Institutional Review Board of S University (approval number 2019-43) granted permission to conduct the study.

\subsection{Statistical Analyses}

Data were analyzed using SPSS version 21 for Windows. Inferential statistical analysis was conducted using chi-square tests and one-way ANOVA. Logistic regression was performed to determine independent factors associated with happiness among older adults living alone, older adults living with their spouse, and older adults living with families.

\section{Results}

\subsection{Sample Characteristics}

The sample consisted of a total of 14,687 older adults: 3,059 (20.8\%) living alone, 6,644 (45.3\%) living with a spouse and 4,984 (33.9\%) living with their families (Table 1). There were significant differences in the sociodemographic characteristics of the three groups $(p<.001)$. Among older adults living alone, there were $692(4.7 \%)$ males and 2,367 (16.1\%) females. Among older adults living with only their spouse, there were 3,786 (25.8\%) males and 2,858 (19.4\%) females. Among older adults living with families, there were 1,845 (12.6\%) males and 3,139 (21.4\%) females. The average ages were 74.86 years $( \pm 6.45)$ for older adults living alone, 72.47 years $( \pm 5.50)$ for older adults living with only their spouse, and 73.56 years $( \pm 6.75)$ for older adults living with their families $(p<.001)$. Although the educational category with the highest percentage in all three groups was elementary school graduates, there was a significant difference in educational level between the groups $(p<.001)$. Regarding marital status, 2,360 older adults living alone were widowed and 2,736 older adults living with their families were married, with these having the highest percentages in these two family types $(p<.001)$. Regarding employment status, in all three groups, the highest percentages were for unemployment. In all three groups, the highest percentages were for those not eligible for basic livelihood rights. The monthly average income with the highest proportion was 500,000 to 990,000 Korean won for older adults living alone, 1,000,000 to 1,999,000 Korean won for older adults living with only their spouse, and over 2,000,000 Korean won for older adults living with their families ( $p$ $<.001)$. 


\subsection{Comparison of Variables Between the Three Groups}

The results of the comparisons of depressive symptoms, present health status, satisfaction with socio-physical environment, social support networks, participation in social activities, and happiness in older adults living alone, living with only their spouse, and living with their families are shown in Table 2. Depressive symptoms and present health status were significantly different between the three groups $(\mathrm{p}<.001)$. Satisfaction with the socio-physical environment, trust in neighbors $(\mathrm{p}=.001)$, help from neighbors $(p<.001)$, satisfaction with overall environment safety $(p=.009)$, satisfaction with public transport $(p=.007)$, and satisfaction with medical facilities $(p=.002)$ were significantly different between the three groups. In terms of social support networks, the frequency of contact with family, neighbors, and friends was significantly different between the three groups $(p<.001)$. In terms of participation in social activities, religious activities, friendship activities, recreational/leisure activities, and philanthropic activities were significantly different between the three groups $(\mathrm{p}<.001)$. There was a significant difference in happiness index between the three groups $(\mathrm{p}<.001)$.

Table 2. Comparison of Depressive Symptoms, Present Health Status, Socio-Physical Environment Satisfaction, Social Support Networks, Social Activity Participation, and Happiness ${ }^{1}$

\begin{tabular}{|c|c|c|c|c|c|c|c|c|c|}
\hline \multirow[t]{2}{*}{ Characteristics } & & \multirow[t]{2}{*}{ Categories } & $\begin{array}{l}\text { Living } \\
\text { alone } \\
(\mathrm{n}= \\
3,059)\end{array}$ & \multicolumn{2}{|c|}{$\begin{array}{l}\text { Living } \\
\text { with } \\
\text { spouse } \\
(\mathrm{n}= \\
6,644)\end{array}$} & \multicolumn{2}{|c|}{$\begin{array}{l}\text { Living with } \\
\text { family } \\
(\mathrm{n}=4,984)\end{array}$} & \multirow[t]{2}{*}{$\begin{array}{l}\chi^{2} \text { or } \\
F\end{array}$} & \multirow[t]{2}{*}{$p$} \\
\hline & & & $\%$ & $n$ & $\%$ & $n$ & $\%$ & & \\
\hline \multicolumn{2}{|c|}{ Depressive symptoms $(\mathrm{M} \pm \mathrm{SD})$} & & $\begin{array}{l}12.48 \pm \\
4.29\end{array}$ & $\begin{array}{l}11.20 \\
3.30\end{array}$ & & \multicolumn{2}{|c|}{$11.71 \pm 3.76$} & 127.84 & $4<.001$ \\
\hline \multicolumn{2}{|c|}{ Present health status $(\mathrm{M} \pm \mathrm{SD})$} & & $\begin{array}{l}13.05 \pm \\
2.01\end{array}$ & $\begin{array}{l}13.70 \\
1.80\end{array}$ & & \multicolumn{2}{|c|}{$13.33 \pm 1.99$} & 132.09 & $9<.001$ \\
\hline \multirow{14}{*}{$\begin{array}{l}\text { Satisfaction with } \\
\text { socio-physical } \\
\text { environment }\end{array}$} & \multirow{2}{*}{$\begin{array}{l}\text { Trust in } \\
\text { neighbors }\end{array}$} & No & 9116.6 & 1,754 & 12.6 & 1,38 & 09.9 & \multirow[t]{2}{*}{13.99} & \multirow[t]{2}{*}{.001} \\
\hline & & Yes & $1,97114.2$ & 4,550 & 32.8 & 3,30 & 923.9 & & \\
\hline & \multirow{2}{*}{$\begin{array}{l}\text { Help from } \\
\text { neighbors }\end{array}$} & No & $1,56311.0$ & 2,886 & 20.3 & 2,44 & 717.2 & \multirow[t]{2}{*}{69.97} & \multirow[t]{2}{*}{$<.001$} \\
\hline & & Yes & $1,3929.8$ & 3,561 & 25.1 & 2,36 & 016.6 & & \\
\hline & \multirow{2}{*}{$\begin{array}{l}\text { Satisfaction } \\
\text { with overall } \\
\text { safety of } \\
\text { environment }\end{array}$} & No & $520 \quad 3.6$ & 987 & 6.9 & 805 & 5.6 & \multirow[t]{2}{*}{9.49} & \multirow[t]{2}{*}{.009} \\
\hline & & Yes & $2,46717.1$ & 5,557 & 38.6 & 4,06 & 328.2 & & \\
\hline & \multirow{2}{*}{$\begin{array}{l}\text { Satisfaction } \\
\text { with natural } \\
\text { environment }\end{array}$} & No & $629 \quad 4.3$ & 1,391 & 9.5 & 1,04 & 87.2 & \multirow[t]{2}{*}{0.26} & \multirow[t]{2}{*}{.876} \\
\hline & & Yes & $2,40016.5$ & 5,217 & 35.8 & 3,88 & 426.7 & & \\
\hline & \multirow{2}{*}{$\begin{array}{l}\text { Satisfaction } \\
\text { with living } \\
\text { environment }\end{array}$} & No & $377 \quad 2.6$ & 729 & 5.0 & 561 & 3.8 & \multirow[t]{2}{*}{3.88} & \multirow[t]{2}{*}{.144} \\
\hline & & Yes & $2,66318.3$ & 35,877 & 40.3 & 4,38 & 130.0 & & \\
\hline & \multirow{2}{*}{$\begin{array}{l}\text { Satisfaction } \\
\text { with public } \\
\text { transport }\end{array}$} & No & $497 \quad 3.4$ & 970 & 6.7 & 681 & 4.7 & \multirow[t]{2}{*}{9.84} & \multirow[t]{2}{*}{.007} \\
\hline & & Yes & $2,53617.4$ & 5,635 & 38.7 & 4,24 & 529.1 & & \\
\hline & \multirow{2}{*}{$\begin{array}{l}\text { Satisfaction } \\
\text { with medical } \\
\text { facilities }\end{array}$} & No & $475 \quad 3.3$ & 864 & 5.9 & 661 & 4.5 & 12.05 & .002 \\
\hline & & Yes & $2,55817.6$ & 5,726 & 39.4 & 4,26 & 229.3 & & \\
\hline
\end{tabular}




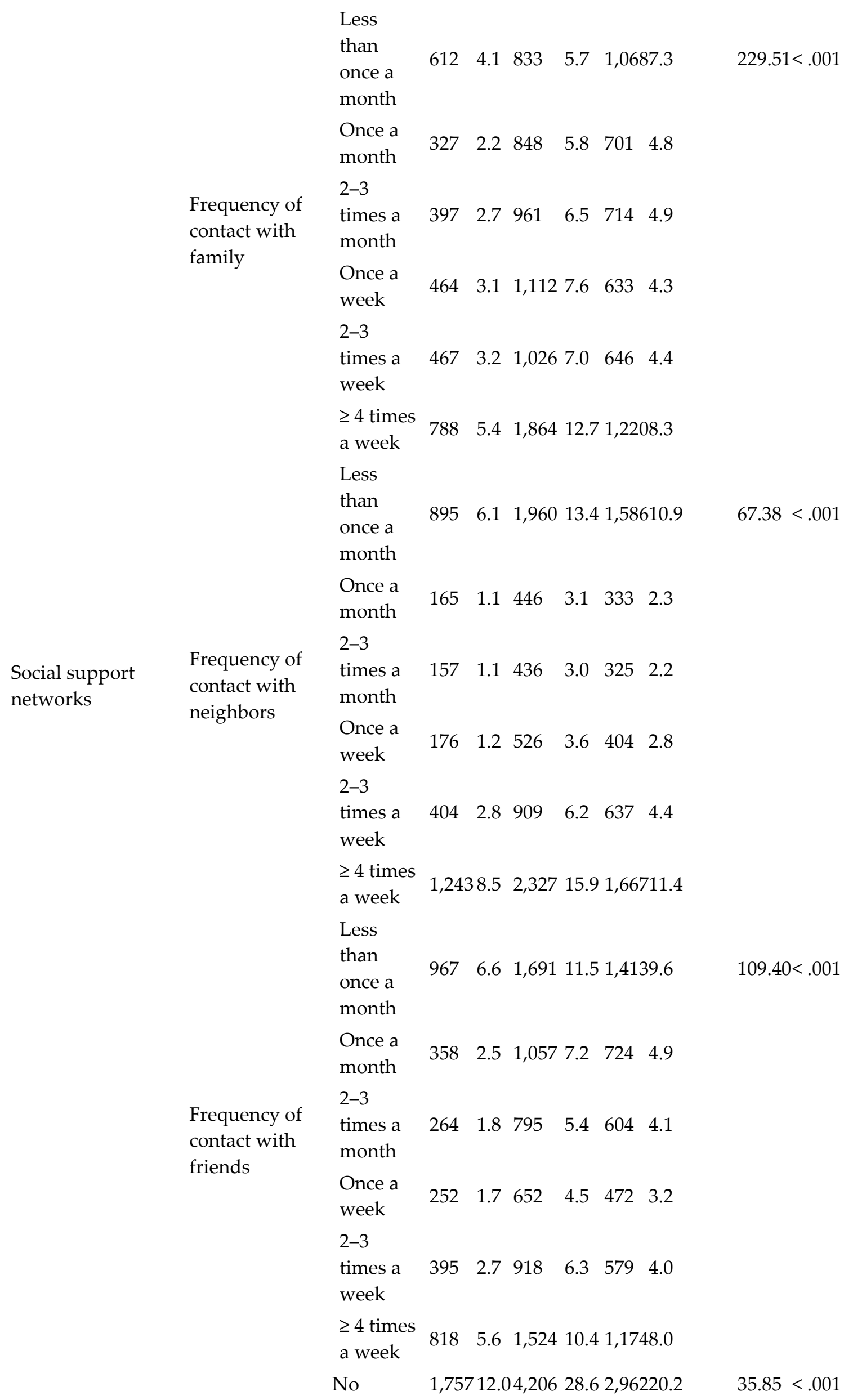




\begin{tabular}{|c|c|c|c|c|}
\hline \multirow{7}{*}{$\begin{array}{l}\text { Participation in } \\
\text { social activities }\end{array}$} & $\begin{array}{l}\text { Religious } \\
\text { activities }\end{array}$ & Yes & $1,3028.92,43816.62,02113.7$ & \\
\hline & Friendship & No & 1,71911.72,557 17.4 2,33015.9 & $275.06<.001$ \\
\hline & activities & Yes & 1,3409.1 4,087 27.8 2,65318.1 & \\
\hline & Recreational/le & No & $2,56317.45,00534.14,00627.3$ & $101.15<.001$ \\
\hline & isure activities & Yes & $\begin{array}{lllll}496 & 3.4 & 1,639 & 11.2976 & 6.6\end{array}$ & \\
\hline & Philanthropic & No & 2,904 19.86,16342.0 4,67931.8 & $17.75<.001$ \\
\hline & activities & Yes & $\begin{array}{llllll}155 & 1.0 & 481 & 3.3 & 303 & 2.1\end{array}$ & \\
\hline Happiness & & & $\begin{array}{l}6.22 \pm \\
2.11\end{array} \quad 6.76 \pm 1.996 .46 \pm 1.94$ & $88.69<.001$ \\
\hline
\end{tabular}

Note. ${ }^{1}$ No responses were excluded

\subsection{Logistic Regression Analyses}

The logistic regression results of general characteristics, depressive symptoms, present health status, satisfaction with socio-physical environment, social support networks, participation in social activities, and happiness of older adults living alone, living with only their spouse, and living with their families are shown in Table 3. The model was constructed with the happiness of older adults as the dependent variable, and with sociodemographic characteristics, depressive symptoms, present health status, satisfaction with socio-physical environment, social support networks, and participation in social activities as independent variables. For each of the three older adult groups, three models were derived. Older adults living alone $(-2 \log L=3100.816$, chi-square $=643.169, p<$ $.001)$, older adults living with only their spouse $(-2 \log L=6275.436$, chi-square $=1140.529, p<.001)$, and older adults living with their families $(-2 \log \mathrm{L}=4914.185$, chi-square $=842.456, \mathrm{p}<.001)$ met the convergence criterion for logistic regression.

Table 3. Logistic Regression Model for Happiness Comparing Three Family Types of Older Adults

\begin{tabular}{|c|c|c|c|c|c|c|}
\hline \multirow{2}{*}{ Variables } & \multicolumn{2}{|c|}{ Living alone } & \multicolumn{2}{|c|}{ Living with spouse } & \multicolumn{2}{|c|}{ Living with family } \\
\hline & OR & $95 \%$ CI & OR & $95 \%$ CI & OR & $95 \% \mathrm{CI}$ \\
\hline Gender & 1.39 & $1.08-1.78$ & 1.06 & $0.92-1.22$ & 1.39 & $1.16-1.66$ \\
\hline Age & 1.01 & $1.00-1.03$ & 1.02 & $1.01-1.03$ & 1.02 & $1.01-1.03$ \\
\hline \multicolumn{7}{|l|}{ Educational level } \\
\hline None & 0.64 & $0.41-1.00$ & 0.62 & $0.45-0.87$ & 0.46 & $0.33-0.65$ \\
\hline Elementary & 0.85 & $0.57-1.26$ & 0.63 & $0.50-0.80$ & 0.57 & $0.43-0.77$ \\
\hline Middle & 0.63 & $0.42-0.95$ & 0.55 & $0.44-0.70$ & 0.61 & $0.45-0.82$ \\
\hline High & 0.72 & $0.48-1.09$ & 0.73 & $0.58-0.92$ & 0.61 & $0.45-0.82$ \\
\hline College or higher & \multicolumn{2}{|l|}{ referent } & \multicolumn{2}{|l|}{ referent } & \multicolumn{2}{|l|}{ referent } \\
\hline \multicolumn{7}{|l|}{ Marital status } \\
\hline Married & 0.90 & $0.47-1.75$ & 1.64 & $0.08-31.79$ & 0.48 & $0.19-1.22$ \\
\hline Divorced & 1.05 & $0.59-1.87$ & 0.91 & $0.04-21.87$ & 0.29 & $0.11-0.78$ \\
\hline Widowed & 1.18 & $0.68-2.07$ & 1.00 & $0.04-24.11$ & 0.50 & $0.19-1.28$ \\
\hline Never married & referent & & referent & & referent & \\
\hline Employment status & 0.74 & $0.59-0.92$ & 0.90 & $0.78-1.04$ & 0.95 & $0.80-1.13$ \\
\hline \multicolumn{7}{|c|}{$\begin{array}{l}\text { Eligibility for basic livelihood } \\
\text { rights }\end{array}$} \\
\hline Yes & 0.68 & $0.53-0.88$ & 1.00 & $0.74-1.34$ & 0.83 & $0.61-1.13$ \\
\hline In the past & 0.82 & $0.40-1.68$ & 1.23 & $0.51-2.93$ & 0.88 & $0.50-1.56$ \\
\hline No & referent & & referent & & referent & \\
\hline \multicolumn{7}{|c|}{ Monthly income $(10,000 \text { won })^{1}$} \\
\hline$<50$ & 0.48 & $0.32-0.72$ & 0.37 & $0.28-0.47$ & 0.53 & $0.36-0.78$ \\
\hline 50-99 & 0.77 & $0.52-1.14$ & 0.56 & $0.46-0.67$ & 0.63 & $0.51-0.79$ \\
\hline
\end{tabular}




\begin{tabular}{|c|c|c|c|c|c|c|}
\hline $\begin{array}{l}100-199 \\
\geq 200\end{array}$ & $\begin{array}{l}0.81 \\
\text { referent }\end{array}$ & $0.53-1.24$ & $\begin{array}{l}0.64 \\
\text { referent }\end{array}$ & $0.54-0.76$ & $\begin{array}{l}0.69 \\
\text { referent }\end{array}$ & $0.58-0.82$ \\
\hline Depressive symptoms & 0.87 & $0.85-0.90$ & 0.87 & $0.85-0.89$ & 0.87 & $0.85-0.89$ \\
\hline Present health status & 1.21 & $1.15-1.28$ & 1.26 & $1.21-1.31$ & 1.23 & $1.18-1.29$ \\
\hline Trust in neighbors & 1.45 & $1.16-1.82$ & 1.32 & $1.13-1.54$ & 1.19 & $1.01-1.42$ \\
\hline Help from neighbors & 0.88 & $0.71-1.09$ & 1.10 & $0.95-1.27$ & 1.11 & $0.95-1.31$ \\
\hline \multicolumn{2}{|c|}{$\begin{array}{l}\text { Satisfaction with overall safety } 1.24 \\
\text { of environment }\end{array}$} & $0.96-1.60$ & 1.04 & $0.87-1.26$ & 0.94 & $0.77-1.16$ \\
\hline \multicolumn{2}{|c|}{ 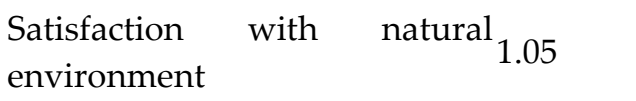 } & $0.83-1.34$ & 1.02 & $0.86-1.20$ & 1.32 & $1.09-1.59$ \\
\hline $\begin{array}{l}\text { Satisfaction with } \\
\text { environment }\end{array}$ & living $_{1.01}$ & $0.76-1.36$ & 1.14 & $0.93-1.41$ & 1.23 & $0.97-1.56$ \\
\hline $\begin{array}{l}\text { Satisfaction with } \\
\text { transport }\end{array}$ & public $_{1.01}$ & $0.77-1.32$ & 1.24 & $1.03-1.50$ & 1.22 & $0.97-1.53$ \\
\hline $\begin{array}{l}\text { Satisfaction with } \\
\text { services }\end{array}$ & medical 1.16 & $0.88-1.53$ & 1.04 & $0.85-1.27$ & 0.92 & $0.73-1.16$ \\
\hline \multicolumn{7}{|l|}{$\begin{array}{l}\text { Frequency of contact with } \\
\text { family }\end{array}$} \\
\hline Less than once a month & 0.92 & $0.70-1.21$ & 0.99 & $0.81-1.22$ & 0.80 & $0.65-0.99$ \\
\hline Once a month & 0.70 & $0.51-0.95$ & 0.84 & $0.68-1.02$ & 0.97 & $0.77-1.22$ \\
\hline 2-3 times a month & 0.87 & $0.64-1.18$ & 1.07 & $0.87-1.31$ & 0.92 & $0.73-1.15$ \\
\hline Once a week & 0.79 & $0.60-1.05$ & 1.17 & $0.97-1.42$ & 1.06 & $0.83-1.35$ \\
\hline $\begin{array}{l}2-3 \text { times a week } \\
\geq 4 \text { times a week }\end{array}$ & $\begin{array}{l}0.75 \\
\text { referent }\end{array}$ & $0.57-0.99$ & $\begin{array}{l}1.03 \\
\text { referent }\end{array}$ & $0.84-1.25$ & $\begin{array}{l}0.97 \\
\text { referent }\end{array}$ & $0.77-1.24$ \\
\hline \multicolumn{7}{|c|}{$\begin{array}{l}\text { Frequency of contact with } \\
\text { neighbors }\end{array}$} \\
\hline Less than once a month & 0.90 & $0.70-1.15$ & 0.83 & $0.69-0.99$ & 1.04 & $0.86-1.27$ \\
\hline Once a month & 0.93 & $0.62-1.38$ & 0.81 & $0.63-1.05$ & 1.35 & $1.00-1.83$ \\
\hline 2-3 times a month & 1.38 & $0.89-2.13$ & 1.13 & $0.86-1.48$ & 1.62 & $1.18-2.22$ \\
\hline Once a week & 0.62 & $0.42-0.93$ & 0.88 & $0.68-1.12$ & 0.85 & $0.65-1.12$ \\
\hline $\begin{array}{l}2-3 \text { times a week } \\
\geq 4 \text { times a week }\end{array}$ & $\begin{array}{l}1.01 \\
\text { referent }\end{array}$ & $0.76-1.34$ & $\begin{array}{l}1.12 \\
\text { referent }\end{array}$ & $0.91-1.37$ & $\begin{array}{l}1.07 \\
\text { referent }\end{array}$ & $0.85-1.35$ \\
\hline \multicolumn{7}{|l|}{$\begin{array}{l}\text { Frequency of contact with } \\
\text { friends }\end{array}$} \\
\hline Less than once a month & 0.81 & $0.63-1.04$ & 0.84 & $0.69-1.02$ & 0.90 & $0.73-1.12$ \\
\hline Once a month & 0.93 & $0.68-1.26$ & 0.69 & $0.56-0.84$ & 0.91 & $0.72-1.15$ \\
\hline 2-3 times a month & 0.90 & $0.64-1.27$ & 0.80 & $0.64-1.01$ & 1.10 & $0.85-1.41$ \\
\hline Once a week & 1.18 & $0.83-1.69$ & 0.81 & $0.63-1.04$ & 1.32 & $0.99-1.75$ \\
\hline $\begin{array}{l}2-3 \text { times a week } \\
\geq 4 \text { times a week }\end{array}$ & $\begin{array}{l}1.44 \\
\text { referent }\end{array}$ & $1.05-1.96$ & $\begin{array}{l}0.92 \\
\text { referent }\end{array}$ & $0.73-1.15$ & $\begin{array}{l}1.19 \\
\text { referent }\end{array}$ & $0.92-1.54$ \\
\hline Religious activities & 1.49 & $1.24-1.79$ & 1.22 & $1.07-1.40$ & 1.31 & $1.13-1.51$ \\
\hline Friendship activities & 1.25 & $1.03-1.52$ & 1.20 & $1.05-1.39$ & 1.08 & $0.92-1.27$ \\
\hline Recreational/leisure activities & 1.38 & $1.05-1.80$ & 1.47 & $1.24-1.75$ & 1.37 & $1.12-1.68$ \\
\hline Philanthropic activities & 1.08 & $0.70-1.67$ & 1.22 & $0.91-1.62$ & 1.20 & $0.86-1.68$ \\
\hline Constant & 0.04 & & 0.02 & & 0.03 & \\
\hline
\end{tabular}

Note. $^{1} 1$ USD = approximately 1,200 Korean won. $\mathrm{OR}=$ odds ratio. $\mathrm{CI}=$ confidence interval.

Significant factors regarding happiness for the three groups were as follows. Among older adults living alone, females were 1.39 times more likely to be satisfied with life than males (odds ratio (OR) $=1.39,95 \%$ confidence interval $(\mathrm{CI})=1.08-1.78)$. Compared to college graduates and above, happiness was found to be $63 \%$ lower in middle school graduates $(\mathrm{OR}=0.63,95 \% \mathrm{CI}=0.42-0.95)$. Compared to 
unemployed older adults living alone, happiness was found to be $74 \%$ lower in the employed (OR $=$ $0.74,95 \% \mathrm{CI}=0.59-0.92)$. Compared to subjects who were not eligible for basic livelihood rights, happiness was $68 \%$ lower in those who were eligible for basic livelihood rights $(\mathrm{OR}=0.68,95 \% \mathrm{CI}=$ $0.53-0.88)$. Compared to those with monthly incomes over 2,000,000 Korean won, happiness was $48 \%$ lower in those with monthly incomes less than 500,000 won (OR $=0.48,95 \% \mathrm{CI}=0.32-0.72)$. As depressive symptoms increased, happiness decreased proportionally $(\mathrm{OR}=0.87,95 \% \mathrm{CI}=0.85-0.90)$. As present health status level increased, happiness also increased proportionally ( $\mathrm{OR}=1.21,95 \% \mathrm{CI}$ $=1.15-1.28)$. Subjects who trusted their neighbors were 1.45 times more likely to be happy than those who did not $(\mathrm{OR}=1.45,95 \% \mathrm{CI}=1.16-1.82)$. Compared to those who had contact with their families four or more times a week, happiness was 70\% lower in subjects who had contact with their families once a month $(\mathrm{OR}=0.70,95 \% \mathrm{CI}=0.51-0.95)$ and $75 \%$ lower in subjects who had contact with their families two to three times a week $(\mathrm{OR}=0.75,95 \% \mathrm{CI}=0.57-0.99)$. Compared to those who had contact with their neighbors four or more times a week, happiness was $62 \%$ lower in subjects who had contact with their neighbors once a month $(\mathrm{OR}=0.62,95 \% \mathrm{CI}=0.42-0.93)$. Compared to those who had contact with friends four or more times a week, happiness was 1.44 times higher in subjects who had contact with friends two to three times a week (OR $=1.44,95 \% \mathrm{CI}=1.05-1.96)$. Subjects who performed regular religious activities were 1.49 times more likely to be happy than those who did not $(\mathrm{OR}=1.49,95 \% \mathrm{CI}=1.24-1.79)$. Subjects who engaged in regular activities with friends were 1.25 times more likely to be happy than those who did not $(\mathrm{OR}=1.25,95 \% \mathrm{CI}=1.03-1.52)$. Subjects who engaged in regular recreational or leisure activities were 1.38 times more likely to be happy than those who did not $(\mathrm{OR}=1.38,95 \% \mathrm{CI}=1.05-1.80)$.

Among older adults living with only their spouse, as age increased, the probability of being satisfied with life increased proportionally $(\mathrm{OR}=1.02,95 \% \mathrm{CI}=1.01-1.03)$. Compared to college graduates and above, all subjects with below high school graduation were less likely to be happy (OR $=0.73,95 \% \mathrm{CI}=0.58-0.92$ ). Compared to subjects with monthly incomes over 2,000,000 Korean won, all those with monthly incomes below $1,990,000$ Korean won were less likely to be happy $(\mathrm{OR}=0.64$, $95 \% \mathrm{CI}=0.54-0.76)$. As depressive symptoms increased, happiness decreased proportionally (OR = $0.87,95 \% \mathrm{CI}=0.85-0.89)$. As present health status level increased, happiness also increased proportionally $(\mathrm{OR}=1.26,95 \% \mathrm{CI}=1.21-1.31)$. Subjects who trusted their neighbors were 1.32 times more likely to be happy than those who did not $(\mathrm{OR}=1.32,95 \% \mathrm{CI}=1.13-1.54)$. Subjects who were satisfied with public transport were 1.24 times more likely to be happy than those who were not (OR $=1.24,95 \% \mathrm{CI}=1.03-1.50$ ). Compared to those who had contact with neighbors four or more times a week, happiness was $83 \%$ lower in subjects who had contact with neighbors less than once a month $(\mathrm{OR}=0.83,95 \% \mathrm{CI}=0.69-0.99)$. Compared to those who had contact with friends four or more times a week, happiness was $69 \%$ lower in subjects who had contact with neighbors less than once a month $(\mathrm{OR}=0.69,95 \% \mathrm{CI}=0.56-0.84)$. Subjects who performed regular religious activities were 1.22 times more likely to be happy than those who $\operatorname{did}$ not $(\mathrm{OR}=1.22,95 \% \mathrm{CI}=1.07-1.40)$. Subjects who engaged in regular activities with friends were 1.20 times more likely to be happy than those who did not (OR $=1.20,95 \% \mathrm{CI}=1.05-1.39$ ). Subjects who engaged in regular recreational or leisure activities were 1.47 times more likely to be happy than those who did not $(\mathrm{OR}=1.47,95 \% \mathrm{CI}=1.24-1.75)$.

Among older adults living with their families, females were 1.39 times more likely to be happy than males $(\mathrm{OR}=1.39,95 \% \mathrm{CI}=1.16-1.66)$. As age increased, the probability of happiness increased proportionally $(\mathrm{OR}=1.02,95 \% \mathrm{CI}=1.01-1.03)$. Compared to college graduates and above, subjects with below high school graduation were less likely to be happy ( $\mathrm{OR}=0.61,95 \% \mathrm{CI}=0.45-0.82)$. Compared to never-married subjects, happiness was $29 \%$ lower in divorced subjects ( $O R=0.29,95 \%$ $\mathrm{CI}=0.11-0.78$ ). Compared to subjects with monthly incomes over 2,000,000 Korean won, those with monthly incomes below 1,990,000 Korean won were less likely to be happy $(\mathrm{OR}=0.69,95 \% \mathrm{CI}=0.58-$ 0.82 ). As depressive symptoms increased, happiness decreased proportionally ( $\mathrm{OR}=0.87,95 \% \mathrm{CI}=$ $0.85-0.89$ ). As present health status level increased, happiness also increased proportionally (OR = $1.23,95 \% \mathrm{CI}=1.18-1.29)$. Subjects who trusted their neighbors were 1.19 times more likely to be happy than those who did not $(\mathrm{OR}=1.19,95 \% \mathrm{CI}=1.01-1.42)$. Subjects who were satisfied with the natural environment were 1.32 times more likely to be happy than those who were not $(\mathrm{OR}=1.32,95 \% \mathrm{CI}=$ 1.09-1.59). Compared to those who had contact with family four or more times a week, happiness 
was $80 \%$ lower in subjects who had contact with family less than once a month $(\mathrm{OR}=0.80,95 \% \mathrm{CI}=$ 0.65-0.99). Compared to those who had contact with neighbors four or more times a week, happiness was 1.62 times higher in subjects who had contact with neighbors two to three times a week $(\mathrm{OR}=$ $1.62,95 \% \mathrm{CI}=1.18-2.22$ ). Subjects who performed regular religious activities were 1.31 times more likely to be happy than those who did not $(\mathrm{OR}=1.31,95 \% \mathrm{CI}=1.13-1.51)$. Subjects who engaged in regular recreational or leisure activities were 1.37 times more likely to be happy than those who did $\operatorname{not}(\mathrm{OR}=1.37,95 \% \mathrm{CI}=1.12-1.68)$.

\section{Discussion}

This study investigated the associations of present health status, depressive symptoms, sociophysical environment, social support networks, and social participation with happiness among older adults living alone, living with only their spouse, and living with their families.

\subsection{Associations of Subject Characteristics, Depression, and Health Status with Happiness}

In this study, the common factors associated with happiness in the three groups were educational level, monthly income, depressive symptoms, and present health status. Several related studies $[3,16,17]$ have indicated that depression and health have a significant effect on the happiness of older adults, and such findings are consistent with our results. In particular, what senior citizens most desire in old age is a healthy life [18]. A previous study [19] found that the lower the educational level of older adults, the poorer their health. This is supported by the results of the present study, in which lower educational levels were associated with reduced happiness. The KIHSA [18] found that economic level had a significant effect on the quality of life in older adults. This finding is supported by our results, which showed that the monthly income of older adults was significantly associated with happiness. Among older adults living alone and living with their families in this study, females were likely to be happier than males. The KIHSA [3] reported that overall quality of life was lower in older females than in older males, which was inconsistent with this study. According to Park [20], females showed higher life satisfaction, with lower expectations for life satisfaction, than males. The results indicated that older females were more likely to be resigned to satisfaction with their lives rather than enjoying a high quality of life. Therefore, it is necessary to make efforts to improve the quality of life of older females.

Among older adults living with only their spouse and older adults living with their families, the higher the age, the higher their happiness. Additionally, divorced subjects were less likely to be happy than never-married subjects. Older adults living alone had the highest depression and the lowest health status. On the contrary, older adults living with only their spouse had the lowest depression and the best overall present health status. According to the KIHSA [18], older adults recognize the importance of their spouses and depend on them. As per that report, the initial two to three years after the death of the spouse is a very challenging time [18]. Another study demonstrated that spouses and other family members had a positive effect on the physical, mental, social, and economic aspects of the lives of older adults [5]. Therefore, the existence of a spouse could be considered one of the most important factors influencing happiness in old age.

Participation in vocational activities was not significantly correlated with happiness in older adults living with only their spouse and older adults living with their families. However, older adults living alone had low levels of happiness when they were working or receiving basic livelihood rights. In the past decade, poverty in older adults living alone has increased dramatically. Moreover, it is reported that the income earned by older adults (labor market, service market, financial market) may be insufficient to escape poverty [21]. This is more likely to be severe in older adults living alone. In this study, among those who answered that their monthly income was more than 2,000,000 Korean won, the proportion of older adults living alone was far lower than those living with only their spouse and those living with their families. It would be hard to find satisfaction and happiness through work because income does not free older adults from poverty.

\subsection{Association of Socio-Physical Environment with Happiness}


In this study, the common aspect of socio-physical environment that was associated with happiness in the three groups was trust in neighbors. This was similar to a previous study [5] in which depression was lower in older adults who had good ties with their neighbors. $\mathrm{Wu}$ and Chan [22] found that living in a public apartment neighborhood and daily participation in public neighborhood events reduced the risk of isolation in older adults. Lee, Cho, Kim, Kim, and Choo [23] stated that residents in a community with strong mutual trust had a higher quality of life than those without trust. This affected health-related quality of life through the creation of a mutually supportive environment, such as belonging to a community, mutual exchange, attachment to neighbors, and so on. The results of this study showed that public transport service conditions were significantly associated with the happiness of older adults living with only their spouse. Therefore, such aspects of the physical environment should be improved continuously. This was similar to previous studies [24] in which the safer the living environment and the lower the car or subway traffic obstacles, the better the subjective and mental health of residents.

\subsection{Association of Social Support Networks with Happiness}

In this study, the common aspect of social support networks that was associated with happiness in the three groups was the frequency of contact with neighbors. The associations of contact frequency with neighbors with happiness differed between the three groups in this study. Older adults living alone and those living with only their spouse had proportionally higher happiness as their neighborhood contact increased. On the contrary, older adults living with their families were shown to be happier if they made proper rather than excessive contact with their neighbors. In older adults living with their families, contact with neighbors or friends did not seem to be significantly associated with happiness. Therefore, this study showed that neighbors are an important support system for older adults living alone and older adults living with only their spouse. Older adults living with only their spouse said that neighbors were very important because they helped them resolve conflicts with their spouses [18].

In this study, the results regarding the frequency of contact with family and friends differed across the three groups. Older adults living alone and those living with their families showed higher happiness as the frequency of contact with family increased. On the contrary, older adults living with only their spouse were found to have higher happiness when their contact with friends was higher. While older adults living with their families were dependent on their children for care, older adult couples were characterized by their independence in their relationships with their children and lived active and less dependent lives [18]. A previous study found that among older adults living with their families, family support and friends' support had a significant effect on self-esteem, whereas among older adults living alone, only family support had a significant effect on self-esteem [25], which is consistent with the results of this study. Another study [26] found that in later life, the relationship with children is difficult to replace with any social relationships, but older adults living alone reduce loneliness through their relationships with neighbors, relatives, and religious activities. $\mathrm{Wu}$ and Chan [22] stated that if older adults living alone in an apartment interacted with other residents and continued to participate in community events, they showed a decrease in loneliness. In this study, in older adults living alone, family and neighborhood contact were significantly correlated with happiness, which was consistent with previous studies. With aging, social networks shrink, and as aging progresses, mobility decreases [18]. In this study, the average age of older adults living alone was highest among the three groups; their spouses were likely to have died, leaving them alone as they aged. Therefore, more efforts are needed to improve the social support network for elderly people living alone compared to those living with only their spouse or their families. 


\subsection{Association of Social Activities with Happiness}

In this study, the common aspects of participation in social activities that were associated with happiness in the three groups were religious activities and recreation or leisure activities. Religious meetings and group activities have a significant effect on the life satisfaction of older adults living alone [17]. In a previous study, older adults who had a religion showed greater happiness than those who did not [3], which is consistent with this study. Older adults seemed to regard fellow church members as family members and important people in their lives because of more frequent contact with them through religious activities than with distant families or relatives.

The results showed that participation in recreational or leisure activities in older adults was positively associated with happiness. This finding is consistent with the results of a previous study [27], which found that older adults experience social satisfaction and resolve social isolation by participating in various group activities. Further, it has been stated that the number of friends in their social network had a significant positive effect on the life satisfaction of older adults. This finding shows that older adults experience healthy feelings through social exchanges with those who have experienced similar life processes, which improves their quality of life [21]. Wu and Chan [22] stated that, in older adults living alone in large cities, contact with friends was more effective in reducing loneliness than contact with relatives who had no ties with them. However, if older adults have limited physical functioning, bonding with adult children has been found to be very important. Therefore, if older adults living alone have no physical limitations and are healthy, socializing with friends and leisure activities are considered to significantly improve subjective health and happiness. In a previous study [28], physical activity in older adults was positively associated with their psychological well-being.

In this study, older adults living alone had contact with friends two or three times weekly, and the more social activities they had, the higher their happiness. This seems to favor the idea of contact with friends based on activities with a purpose rather than simply meeting friends without purpose. A previous study [27] showed that productive leisure activities, such as exercise, volunteer work, and travel in older adults living alone had a positive effect on their physical and mental health. Older adults who participated in various programs at senior welfare centers reported that their happiness improved [29] and depression decreased significantly [30]. These findings suggest that continued provision of and participation in social activities for older adults, especially those living alone, may reduce depression and improve happiness. Therefore, governments and communities should continue to develop and provide social and leisure programs for older adults, especially those living alone, to improve their health and happiness.

The overall results of this study showed interesting differences between the three groups. While the physical environment was significantly associated with happiness among older adults living with only their spouse and older adults living with their families, it was not so for older adults living alone. In social support networks, contact with family and neighbors in older adults living alone, contact with neighbors and friends in older adults living with only their spouse, and contact with family in older adults living with their families were significantly associated with happiness. In social activity participation, a significant correlation with happiness was found for all three groups, although this did not apply to philanthropic activities. Considering these characteristics, there is a need for happiness promotion programs for older adults that take family type into consideration.

\subsection{Limitations}

This study had several limitations. All the secondary data collected were cross-sectional, making it difficult to make causal inferences. Those who were depressed may have been more likely to live alone. Older adults living alone were likely to have lower incomes than those living with their families because of the small number of family members. In old age, income has a big impact on the quality of life [3]. Attempts to generalize the results of this study, which were obtained using secondary data originally collected for another purpose, must be undertaken with caution. The data acquired had inherent limitations. For example, we did not have information on whether subjects 
had a history of major depression. The data used in this study were collected using a self-report. Thus, the possibility of response bias cannot be eliminated. Finally, the data were obtained through the 2017 Korean Community Health Survey organized by the KCDC. While the data were collected at the same time across the country, with the data collectors receiving adequate information about the survey in advance, there may have been differences in how they conducted the surveys.

\subsection{Implications for Further Research}

There is a need for continuing studies that consider subject characteristics, for example, the severity of depression or other diseases. Additionally, we suggest the need for intervention studies that show the mediating effect of happiness improvement programs using the factors associated with happiness in older adults identified in this study.

\section{Conclusions}

This study aimed to compare the associations of socio-physical environment, social support networks, and social activities with the happiness of older adults living alone, with only their spouses, and with their families. The group-wise results are summarized as follows.

Among older adults living alone, female gender, higher educational levels, economic ability to survive without working, absence of depression, good health status, more frequent contact with family and trusted neighbors, contact with friends two or three times a week, and regular participation in religious, friendship, and recreational or leisure activities were the factors associated with a higher likelihood of happiness. Among older adults living with only their spouse, older age, higher educational levels, economic ability to survive without working, absence of depression, good health status, convenience in using public transportation, more frequent contact with trusted neighbors and friends, and regular participation in religious, friendship, and recreational/leisure activities were the factors associated with a higher likelihood of happiness. Among older adults living with their families, older age, never having been divorced, higher educational levels, economic ability to survive without working, absence of depression, good health status, more frequent contact with family, contact with trusted neighbors two or three times a week, and regular participation in religious and recreational/leisure activities were the factors associated with a higher likelihood of happiness.

The family is an essential support system for older adults. It was confirmed that there were differences in the factors associated with older adults' happiness according to family type. Therefore, attempts to ensure the happiness of older adults must adequately account for their various circumstances, including family type. Governments and communities should improve the sociophysical environment for older adults, and continue to develop and provide social activity programs to improve their health and happiness considering various individual characteristics according to family type.

Author Contributions: Conceptualization, EJW; methodology, EJW, IOS; software, EJW, IOS ; validation, EJW ; formal analysis, EJW ; investigation, EJW; resources, EJW; data curation, EJW; writing-original draft preparation, EJW, IOS; writing - review and editing, EJW, IOS; visualization, EJW, IOS; supervision, EJW; project administration, EJW, IOS; funding acquisition, EJW, IOS. All authors have read and agreed to the published version of the manuscript.

Funding: This research was funded by the Sehan University Research Fund in 2020.

Acknowledgments: We are grateful to the Korea Centers for Disease Control and Prevention (KCDC)to allowing the materials to be used and to Sehan University for funding this research.

Conflicts of Interest: The authors declare no conflict of interest.

\section{References}

1. Korean National Statistical Office. KOSIS aged population. Available online: http://kosis.kr/statHtml/statHtml.do?orgId=101\&tblId=DT_1YL20631\&vw_cd=MT_GTITLE01\&list_id=10 
1\&seqNo=\&lang_mode=ko\&language=kor\&obj_var_id=\&itm_id=\&conn_path=MT_GTITLE01 (accessed on 5 August 2019)

2. Korean National Statistical Office. Prospective estimating population and household. Available online: http://www.index.go.kr/unify/idx-info.do?idxCd=4034 (accessed on 5 August 2019)

3. Korea Institute for Health and Social Affairs. Development and application of a quality of life indicator system for older persons. Available online:

https://www.kihasa.re.kr/web/publication/research/view.do?menuId=44\&tid=71\&bid=12\&division=001\& keyField=title\&searchStat=2019\&key=\%EC\%82\%B6\%EC\%9D\%98+\%EC\%A7\%88\&ano=2237 (accessed on 10 October 2019)

4. Cohen-Mansfield, J.; Hazan, H.; Lerman, Y.; Shalom, V. Correlates and predictors of loneliness in olderadults: A review of quantitative results informed by qualitative insights. Int Psychogeriatr 2016, 28, 557-576, doi: 10.1017/S1041610215001532

5. Yoon, J.A.; Kang, J.S.; Bae, M.J.; Lee, N.Y.; Lee, C.M.; Jeon, W.J. Comparison of psychological well-being, self-esteem and satisfaction on life between the elderly living alone and elderly living with other. Journal of the Korea Advanced Institute of Industrial Economics and Trade 2016, 8, 63-69.

6. Lee, S.S.; Kim, S.H. A study on the living conditions and social welfare needs of the elderly who lives alone in the farming and fishing communities-focused on the province of Jeollanamdo. Journal of Korean Regional Development 2011, 11, 23-60.

7. Korean Ministry of Government Legislation. Constitution. Available online: http://www.moleg.go.kr (accessed on 15 August 2019)

8. Easterlin, R.A. Does economic growth improve the human lot? Some empirical evidence. In Nations and Households in Economic Growth: Essays in Honor of Moses Abramovitz, David P.A., Reder M.W., Eds.; Academic Press: New York, USA, 1974; pp. 89-125.

9. Organization for Economic Cooperation and Development. Better life index. Available online: http://www.oecdbetterlifeindex.org (accessed on 15 October 2019)

10. Korea Institute for Health and Social Affairs. Korea happiness index. Available online: https://www.kihasa.re.kr/web/publication/research/view.do?menuId=44\&tid=71\&bid=12\&division=001\& keyField=title\&searchStat=2019\&key=\%ED\%96\%89\%EB\%B3\%B5\%EC\%A7\%80\%EC\%88\%98\&ano=2333 (accessed on 10 October 2019)

11. United Nations. Political declaration and Madrid International Plan of Action on Ageing: United Nations Second World Assembly on Ageing. Available online:

https://www.un.org/esa/socdev/documents/ageing/MIPAA/political-declaration-en.pdf (accessed on 15 October 2019)

12. World Health Organization. Global age-friendly cities: A guide. Available online: https://www.who.int/ageing/publications/Global_age_friendly_cities_Guide_English.pdf (accessed on 15 October 2019)

13. Green, L.W.; Kreuter, M.W. Health Program Planning: An Educational and Environmental Approach, 2nd ed.; Mayfield Publishing Company: California, USA, 1991.

14. Yamada, T.; Chen, C.; Murata, C.; Hirai, H.; Ojima, T.; Kondo, K.; Harris, J.R. Access disparity and health inequality of elderly: Unmet needs and delayed healthcare. Int J Environ Res Public Health 2015, 12, 17451772, doi: 10.3390/ijerph120201745

15. Green, L.W.; Kreuter, M.W. Health Program Planning: An Educational and Ecological Approach, 4th ed.; McGraw-Hill: New York, USA, 2005.

16. Choi, M.Y.; Kwak, H.K.; Park, H.S. The effect of community social capital on the elderly's subjective quality of life: Focusing on the mediating effect of the elderly depression. J Welf Aged 2014, 66, 307-328.

17. Moon, J.H.; Kim, D. Factors influencing life satisfaction in elderly living alone. The Journal of the Korea Contents Association 2018, 18, 44-54, doi: 10.5392/JKCA.2018.18.01.044

18. Korea Institute for Health and Social Affairs. Living profiles of older-persons-only households and policy implications. Available online:

https://www.kihasa.re.kr/web/publication/research/view.do?menuId=44\&tid=71\&bid=12\&division=001\& keyField=title\&searchStat=2019\&key=\%EB\%8B \%A8\%EB\%8F\%85\%EA\%B0\%80\%EA\%B5\%AC\&ano=1788 (accessed on 10 October 2019)

19. Korean Ministry of Health and Welfare. 2015 national survey report on older persons. Available online: http://www.1661-2129.or.kr (accessed on 15 October 2019)

20. Park, K.N. Gender differences in the life satisfaction of elderly. J Korean Geriatr Soc 2004, 24, 13-29. 
21. Lim, J.R. Phenomenological research on the experience of melancholy elderly women: Focusing on the friend-making program of elderly people living alone. Korean J Gerontol Social Welf 2016, 71, 9-33.

22. Wu, T.; Chan, A. Families, friends and the neighborhood of older adults: Evidence from public housing in Singapore. J Aging Res 2011, 2012, 1-7, doi: 10.1155/2012/659806

23. Lee, H. K.; Cho, S. H.; Kim, J. H.; Kim, Y. K.; Choo, H. I. Influence of self efficacy, social support and sense of community on health-related quality of life for middle-aged and elderly residents living in a rural community. J Korean Acad Nurs 2014, 44, 608-616, doi: 10.4040/jkan.2014.44.6.608

24. Putrik, P.; Vries, N.K.; Mujakovic, S.; Amelsvoort, L.; Kant, I.; Kunst, A. E.; van Oers H.; Jansen, M. Living environment matters: Relationships between neighborhood characteristics and health of the residents in a Dutch municipality. J Community Health 2015, 40, 47-56, doi: 10.1007/s10900-014-9894-y

25. Seo, I.K.; Lee, Y.S. An exploratory study on the influence of social support and community environment on elderly self-esteem: A comparative study on people who live alone and those who live with family. The Korean Journal of Local Government \& Administration Studies 2014, 28, 371-399.

26. Sung, M.H.; Lim, Y.M.; Joo, K.S. The relationship between social support and loneliness in elderly women living alone. J Korean Public Health Nurs 2011, 25, 95-106.

27. Kim, Y.S.; Ha, W.Y. A study of the effect of participation in productive leisure activities on the suicide ideation and physical and mental health of elderly living alone. Health Soc Welf Rev 2015, 35, 344-374, doi: 10.15709/hswr.2015.35.4.344

28. Lee, B.; Howard, E.P. Physical activity and positive psychological well-being attributes among U.S. Latino older adults. J Gerontol Nurs 2019, 45, 44-56, doi: 10.3928/00989134-20190426-01

29. Lee, B.I.; Kim, D.R.; Lim, H.N.; Kim, K.H. A convergence study on the happiness and participating desire of economic activity among elderly living alone. Journal of the Korea Convergence Society 2018, 9, 109-116, doi: 10.15207/JKCS.2018.9.8.109

30. Jang, A.S.; Hwang, E.J. The effects of comprehensive health care program for living alone older people on blood pressure, fasting glucose, body composition, depression at a senior welfare center. J Korea Acad Industr Coop Soc 2017, 18, 526-535, doi: 10.5762/KAIS.2017.18.11.526 